\title{
Injury Difference of the Un-Aggregated and Aggregated Calcium Oxalate Monohydrate on Human Kidney Proximal Tubular Epithelial Cells
}

\author{
Li-shan HUANG, Da GUO and Jian-ming OUYANG ${ }^{a, *}$
}

Department of Chemistry, Jinan University, Guangzhou 510632, China

atoyjm@jnu.edu.cn

${ }^{*}$ Corresponding author

Keywords: Cell Modulation, Calcium Oxalate, Crystal Aggregation, Osteopontin.

\begin{abstract}
Purpose: To study the injury difference of the un-aggregated and aggregated calcium oxalate monohydrate $(\mathrm{COM})$ on human kidney proximal tubular epithelial (HKC) cells, and to discuss the molecular and cellular mechanism of kidney stone formation. Methods: The cell injure by crystals was evaluated by measuring the cell viability, malonaldehyde (MDA) release, and the expression level of osteopontin (OPN). Scanning electron microscope (SEM) was used to observe the HKC-COM crystal adhesion. Results: After adhesion to COM, cell vitality dropped, both MDA release and OPN expression increased. The crystal injury in HKC was in a time-dependent manner within $24 \mathrm{~h}$. aggregated COM caused more serious injury than un-aggregated COM, and the adhesive capacity of aggregated COM was also obviously stronger than un-aggregated COM. Conclusions: Aggregated COM crystals enhanced the risk of calcium oxalate stone formation much stronger than un-aggregated COM crystals.
\end{abstract}

\section{Introduction}

Nephrolithiasis is a common disease around the world. Despite the number of researchers approaching the problem from different angles, the mechanism for the formation of the stones is not yet clearly understood. Various factors, such as oxalate [1], calcium oxalate $(\mathrm{CaOx})$ crystals [2,3], and free radicals like superoxide anion $\left(\mathrm{O}_{2}^{\cdot-}\right)$ [4], were thought to be involved in renal tubular cell injury.

The damaged renal tubules epithelial cells is mediated by lipid peroxidation reaction through the generation of oxygen free radicals. Free radical scavengers, such as catalase and superoxide dismutase (SOD), could provide significant protection against renal tubular cell injury induced by oxalate and $\mathrm{CaOx}$ crystals $[4,5]$. Cultured epithelial cell lines have been used to examine the mechanisms in the renal epithelium leading to urolithiasis despite the clear differences among various cultured epithelial cells.

At present, studies on crystal-cell interactions in vitro focus on two aspects. The first is to investigate the injurious effect of oxalate, $\mathrm{CaOx}$ crystals, and free radicals on cells. For example, Lieske et al. [6] exposed LLC-PK 1 and IMCD cells to oxalate for 15 min-72 h. In response to all oxalate concentrations $(0.5-2.0 \mathrm{mmol} / \mathrm{L})$ and lengths of exposure (15 min-2 h), both cell lines generated reactive oxygen species (ROS). Khan et al. [7] exposed NRK-52E cells in vitro to synthetic and biological $\mathrm{CaOx}$ or brushite crystals. Results indicated that even though both synthetic and naturally produced biocrystals invoke an injurious response from renal epithelial cells, the latter were 
significantly less injurious and inflammatory. Exposure to low concentrations of these crystals alone may not be sufficient to invoke an inflammatory response, cause uncovering of crystal binding molecules on epithelial cell surfaces, and promote crystal attachment and retention. The second approach is to investigate the process of crystal attachment to normal or injurious renal tubular cells. Lieske et al. [8] identified the most prominent COM crystal-binding protein $(\mathrm{Mr}, 37 \mathrm{kD})$ on the surface of MDCKI cells as annexin II (Ax-II). However, the impact of different crystal properties toward renal tubular epithelial cells is still not clear; even the differences between un-aggregated and aggregated known at present in urine have not been reported.

\section{Experimental details}

\section{Reagents and apparatus}

Human kidney tubular epithelial cells (HKC) was purchased from Shanghai Cell Bank, Chinese Academy of Sciences. Cell proliferation assay kit (Cell Couting Kit 8, CCK-8) (Dojindo Laboratory, Kumamoto, Japan). MDA kit and superoxide dismutase kit (Jiancheng Institute of Biotechnology of Nanjing, China). The first antibody of OPN and FITC secondary antibody (SANTA CRUZ, America). Cell culture plate (Iwaki, Japan).

The medium DMEM-F12 and newborn calf serum (HyClone, America). Penicillin and streptomycin (Beijing Pubo Biology Technology limited Co.). 30\% Hydrogen peroxide $\left(\mathrm{H}_{2} \mathrm{O}_{2}\right)$ and other conventional reagents were all analytically pure.

$\mathrm{X}$-L type environmental scanning electron microscope (ESEM, Philips). Laser confocal microscope (LSM510 META DUO SCAN, ZEISS, Germany). Inverted fluorescence microscope (IX51) (Olympus Corporation, Japan). Enzyme mark instrument (Safire2 ${ }^{\mathrm{TM}}$, Tecan, Männedorf, Switzerland).

\section{Preparation of COM Crystals}

Un-aggregated COM crystals: Approximately $0.2 \mathrm{~g}$ of gelatin was added to $20 \mathrm{~mL}$ of distilled water and heated to dissolve completely. Impurities in gelatin were removed via centrifugation using $400 \mathrm{~mL}$ of $0.05 \%$ (W/V) of the gelatin solution and heated to $75^{\circ} \mathrm{C}$. In addition, about $20 \mathrm{~mL}$ of $\mathrm{K}_{2} \mathrm{Ox}(20 \mathrm{mM})$ solution was added and stirred at 300 rpm for $10 \mathrm{~min}$. Afterward, $20 \mathrm{~mL}$ of $\mathrm{CaCl}_{2}(20 \mathrm{mM})$ solution was added, and the speed and reaction was maintained for $10 \mathrm{~min}$. The prepared crystals were collected with the same processing as above.

Aggregated COM crystals: A total of $150 \mathrm{~mL}$ of $\mathrm{Na}_{2} \mathrm{Ox}(10 \mathrm{mM})$ and $150 \mathrm{~mL}$ of $\mathrm{NaCl}$ $(0.5 \mathrm{M})$ were mixed in $500-\mathrm{mL}$ beaker, evenly stirred, and heated to $70^{\circ} \mathrm{C}$. Afterward, $150 \mathrm{~mL}$ of $\mathrm{CaCl}_{2}(5 \mathrm{mM})$ solution preheated to $70^{\circ} \mathrm{C}$ was added in the reaction mixture, and the reaction was maintained at $70^{\circ} \mathrm{C}$ under continuous stirring at $900 \mathrm{rpm}$ for $2 \mathrm{~min}$. The final solution was incubated overnight at room temperature. The prepared crystals were collected with the same processing as the synthesis of COM-elliptical.

\section{Cell Culture}

The cells were cultured in DMEM-F12 supplemented with $10 \%$ newborn calf serum (Gibco), $100 \mathrm{U} / \mathrm{ml}$ penicillin-100 $\mu \mathrm{g} / \mathrm{ml}$ streptomycin antibiotics (Gibco), with $\mathrm{pH} 7.4$ at $37^{\circ} \mathrm{C}$ in $5 \%$ carbon dioxide air atmosphere. Cell culture media were exchanged every two days as needed. Cell viability of HKC cells was evaluated colorimetrically with CCK-8. The cells were divided into two groups: A group was control group, and B 
group was crystal group. Cells in A group were only exposed to serum-free culture medium, while cells in B group were exposed to serum-free culture media containing $200 \mu \mathrm{g} / \mathrm{ml}$ un-aggregated or aggregated COM.

\section{Measurements of malondialdehyde (MDA) content}

The MDA kit was used to measure the content of MDA, which was strictly conducted according to the instructions of kit.

\section{Detection of osteopontin (OPN) expression by HKC during adhesion}

Cell concentrations were adjusted to $2 \times 10^{5} \mathrm{cell} / \mathrm{ml}$. Cell suspension was assimilated and then plated in 12-well plates $(1 \mathrm{ml} /$ well $)$ with cover slips at bottom and cultured in incubator at $37^{\circ} \mathrm{C}$ with $5 \% \mathrm{CO}_{2}$. After $24 \mathrm{~h}$, cells almost covered the whole cover slips. The culture media were removed, and the cells were rinsed once with D-Hanks and then were divided into two groups (A: control group, and B crystal group) as described above.

After cells were incubated with crystals for 2, 6, 12 and $24 \mathrm{~h}$, respectively, they were washed for three times with PBS (5 min each time), fixed with $4 \%$ paraformaldehyde for $10 \mathrm{~min}$, washed with PBS for three times (3 min each time), blocked with sheep serum for $20 \mathrm{~min}$, and incubated with primary antibody of OPN (1:100) overnight at $4^{\circ} \mathrm{C}$. Cells were then rinsed for three times with PBS, incubated with FITC secondary antibody $(1: 100)$ under darkness for $0.5 \mathrm{~h}$ at $37^{\circ} \mathrm{C}$, rinsed three times with PBS again, and stained and sealed with DAPI. The fluorescence was observed using Laser confocal microscopy (LSM). The nucleus was in blue while the OPN was in green.

\section{SEM observation of HKC-COM adhesion before and after the injury}

The cells were incubated in 6 -well plates $\left(4 \times 10^{5}\right.$ cell $/ \mathrm{ml}, 2 \mathrm{ml} /$ well $)$ with cover slips at bottom.

When cells were incubated for 2, 6, 12 and $24 \mathrm{~h}$, respectively, the cover slips were taken out, the culture media were removed, the nonadherent crystals were washed away with D-Hanks, and the cells were fixed with $2.5 \%$ glutaraldehyde at $4^{\circ} \mathrm{C}$ for $24 \mathrm{~h}$. Fixed cells were dehydrated in ethanol gradient $(30 \%, 50 \%, 70 \%, 90 \%$ and $100 \%$, respectively), refixed with isoamyl acetate, then dried under the critical point of $\mathrm{CO}_{2}$, and treated by gold sputtering. Adhesion between cells and crystals were observed by SEM.

\section{Results and Discussion}

\section{Characterization of COM crystals}

The morphologies of aggregated and un-aggregated COM were shown in Figs. 1a and 1b. The un-aggregated COM was mainly hexagon. The dimensions of most crystals ranged from $12 \mu \mathrm{m}$ to $16 \mu \mathrm{m}$, with an average size of $15 \pm 2 \mu \mathrm{m}$. The spectra of XRD (Figs. 1c \& 1d) demonstrated that these crystals were COM, the d values in spectrogram were $0.598,0.365,0.298$ and $0.236 \mathrm{~nm}$, which belong to (101), (020), ( $\overline{2} 02)$ and (130) of the crystal planes of COM, respectively.

\section{The changes of $\mathrm{HKC}$ cell vitality during adhesion}

The nonradioactive property of cell counting kit-8 (CCK-8) assay allows sensitive colorimetricassays for the determination of the number of viable cells in cell 
proliferation and cytotoxicity assays. Detection sensitivity of CCK-8 is higher than other tetrazolium salts, such as MTT, XTT, MTS, or WST-1.

Fig. 2a showed the vitality of HKC cell exposure to aggregated COM and un-aggregated COM crystals for different treatment times. As the adhesive time increased, the cell vitality of HKC in both aggregated COM group and un-aggregated COM group gradually reduced, but the vitality of $\mathrm{HKC}$ decreased more for aggregated COM than the un-aggregated COM $(P<0.05)$. These indicated that COM crystals damaged cells, furthermore, the aggregated COM damaged much stronger, leading to declined cell vitality. For HKC in aggregated COM group, the vitality decreased rapidly in the first $2 \mathrm{~h}$.

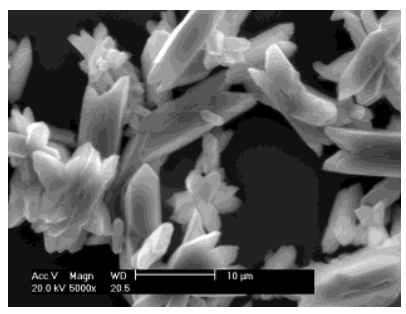

(a)

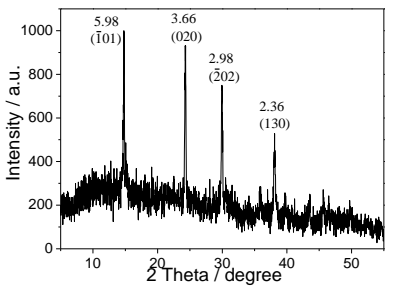

(c)

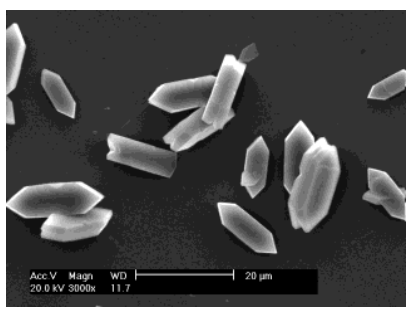

(b)

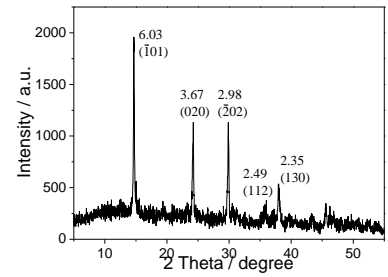

(d)

Fig. 1. Morphology and XRD of un-aggregated or aggregated COM crystals. $(a, c)$ aggregated COM; $(b, d)$ un-aggregated COM. The bars: (a) $10 \mu \mathrm{m}$; (b) $20 \mu \mathrm{m}$.

\section{The change of MDA content during adhesion}

The change of MDA content usually reveals the level of lipid peroxidation in vivo, and indirectly reflects the degree of cell injury. The change of MDA content of HKC cell exposure to aggregated $\mathrm{COM}$ and un-aggregated COM crystals for different treatment times was shown in Fig. 2b, in which we could see:

The MDA content augmented as treatment time increased $(P<0.05)$, indicating the degree of cell injury aggravated with time.

During the treatment process of COM crystals, the MDA content in aggregated COM was generally higher than that un-aggregated COM group, suggesting that the aggregated COM were damaged $\mathrm{HKC}$ more severely.
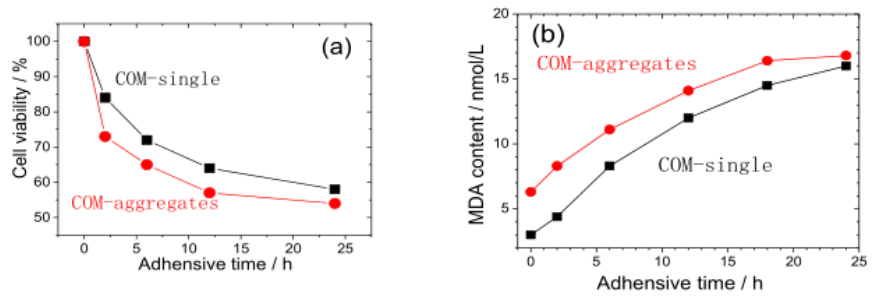

Fig. 2 Cell viability (a) and MDA content (b) assay of HKC cells after exposure to aggregated and un-aggregated COM crystals for different time. Crystal concentration: $200 \mu \mathrm{g} / \mathrm{mL}$. 


\section{Expression of Crystal Adhesion Molecule OPN on HKC Surface}

OPN is a crystal-binding molecule that plays an important role in cell adhesion. OPN expression in HKC cells after treatment with COM crystals is shown in Fig. 3. Only a little green fluorescence could be found around HKC in control group (Fig. 3a). However, the crystal-treated groups showed obvious green fluorescence (Figs. 3b \& 3c). The fluorescence intensity of aggregated COM crystals was obviously higher than that in the un-aggregated COM crystal groups, indicating that aggregated crystals induce higher OPN expression.

OPN was a negatively charged noncollagenous matrix protein with a molecular weight of about $44 \mathrm{kD}$. It had 300 amino-acid residues, among which negatively charged aspartate, serine and glutamic acid had a high proportion ${ }^{[9]}$. Therefore, OPN could not only adsorb the calcium ions in solution, but also adhere to the positively charged COM crystals ${ }^{[10]}$. Indeed, it has been confirmed that OPN has the capability to bind calcium ions, calcium oxalate crystals, and hydroxyapatite in urine ${ }^{[11]}$.

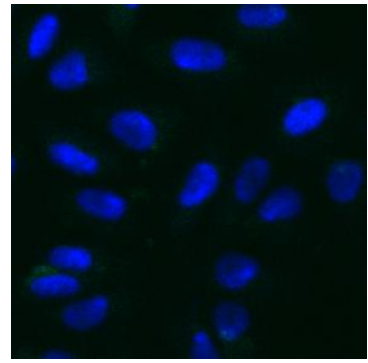

(a)

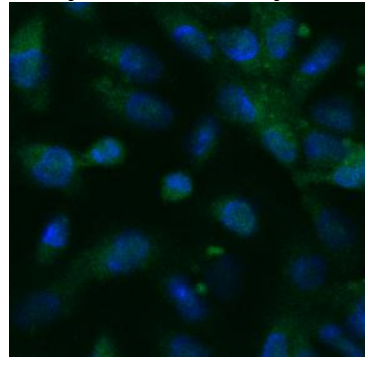

(b)

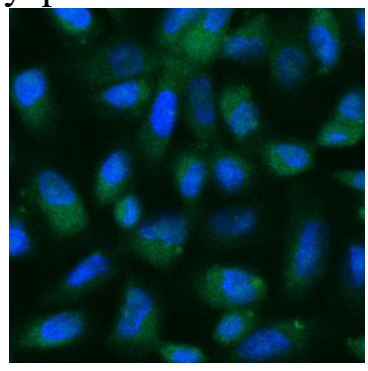

(c)

Fig. 3. Laser scanning confocal microscope images $(\times 630)$ showing fluorescent changes of OPN on HKC cells after exposure to aggregated and un-aggregated COM crystals. a) Control group; (b) COM-single; (c) COM-aggregates. $c(\mathrm{COM})=200 \mu \mathrm{g} / \mathrm{ml}$. The nucleus was in blue and the expressed OPN was in green.

\section{SEM Observation of Adhesion Between COM Crystals and HKC Cells}

The adhesion between HKC cells and COM crystals was detected by SEM (Fig. 4). The morphology of control cells (Fig. 4a) was plump with intact and smooth surface. However, after treatedb with crystals, the HKC shrank with rough surface, and the cell debris were attached on cells, and most of the flagella and synapses of HKC were fractured and shed. It suggested that the cells had been seriously injured by crystals, and the surface and intracellular structure were destroyed, thus producing a large amount of cell debris.

The aggregated COM crystals also presented evident aggregation after these crystals adhered to the cell surface (Fig. 4c). However, the un-aggregated COM crystals were almost individual particles (Fig. 4b).

The above results indicated that the interaction of HKC with COM-aggregates caused further injury to $\mathrm{HKC}$, thus enhancing the risk of kidney stone formation.

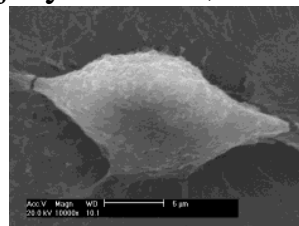

(a)

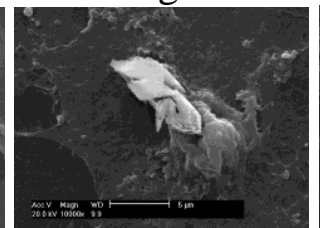

(b)

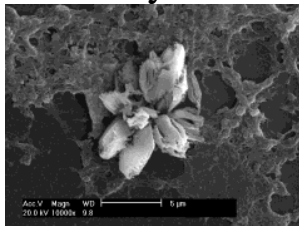

(c)

Fig. 4. SEM images of HKC cells after exposure to aggregated and un-aggregated COM crystals. (a) Normal HKC cells; (b) COM-single; (c) COM-aggregates. c(COM)=200 $\mu \mathrm{g} / \mathrm{ml}$; Incubated time: $12 \mathrm{~h}$. The bars: $5 \mu \mathrm{m}$. 


\section{Conclusions}

Aggregated COM caused more serious injury than un-aggregated COM. The crystal injury in $\mathrm{HKC}$ was in a time-dependent manner within $24 \mathrm{~h}$. The adhesive capacity of aggregated COM was also obviously stronger than un-aggregated COM. After injured by COM crystals, cell vitality dropped, both MDA release and OPN expression inecreased. Aggregated COM crystals enhanced the risk of calcium oxalate stone formation much atronger than un-aggregated COM crystals.

\section{Acknowledgements}

This research work was granted by the Natural Science Foundation of China (No. 81670644).

\section{References}

[1] Kovacevic L, Lu H, Caruso J A, and lakshmanan Y. Renal Tubular Dysfunction in Pediatric Urolithiasis: Proteomic Evidence. Urology, 2016, 92: 100-105.

[2] Narula S, Tandon S, Singh S K, Tandon C. Kidney stone matrix proteins ameliorate calcium oxalate monohydrate induced apoptotic injury to renal epithelial cells. Life Sci., 2016, 164: 23-30.

[3] Tsuji H, Wang W, Sunnil J, Shimizu N, Yoshimura K, Uemura H, Peck A B, khan $\mathrm{S}$ R. Involvement of rennin-angiotensin-aldosterone system in calcium oxalate crystal induced activation of NADPH oxidase and renal cell injury. World J. Urol, 2016, 34(1): 89-95.

[4] Escobar C, Byer KJ, Khaskheli H, Khan SR. Apatite induced renal epithelial injury: insight into the pathogenesis of kidney stones. J. Urol., 2008, 180(1): 379-387.

[5] Rashed T, Menon M, Thamilselvan S. Molecular mechanism of oxalate-induced free radical production and glutathione redox imbalance inrenal epithelial cells: effect of antioxidants. Am. J. Nephrol., 2004, 24(5): 557-568.

[6] Greene EL, Farell G, Yu SH, Matthews T, Kumar V, Lieske JC. Renal cell adaptation to oxalate. Urol. Res., 2005, 33(5): 340-348.

[7] Escobar C, Byer KJ, Khan SR. Naturally produced crystals obtained from kidney stones are less injurious to renal tubular epithelial cells than synthetic crystals. BJU Int., 2007, 100: 891-897.

[8] Kumar V, Farell G, Deganello S, Lieske JC. Annexin II is present on renal epithelial cells and binds calcium oxalate monohydrate crystals. J. Am. Soc. Nephrol., 2003, 14: 289-297.

[9] Ouyang J-M, Yao X-Q, Tan J, Wang F-X. Renal epithelial cell injury and its promoting role in formation of calcium oxalate monohydrate. J. Biol. Inorg. Chem., 2011, 16(3): 405-416.

[10] Evan A P, Coe F L, Rittling S R, Bledsoe S M, Shao Y Z, Lingeman J E, Worcester E M. Apatite plaque particles in inner medulla of kidneys of calcium oxalate stone formers: Osteopontin localization. Kidney Int., 2005, 68: 145-154. 
[11] Verhulst V, Asselman M, Persy V P, Schepers M S J, Helbert M F, Verkoelen C F, De Broe M E. Crystal retention capacity of cells in the human nephron:Involvement of CD44 and its ligands hyaluronic acid and osteopontin in the transition of a crystal binding-into a nonadherent epithelium. J. Am. Soc. Nephrol., 2003(13): 107-115. 